\title{
BMJ Open Cost-effectiveness of telephone coaching for physically inactive ambulatory care hospital patients: economic evaluation alongside the Healthy $4 U$ randomised controlled trial
}

\author{
Stephen Barrett $\left(\mathbb{0},{ }^{1}\right.$ Stephen Begg, ${ }^{2}$ Paul O'Halloran, ${ }^{3}$ Michael Kingsley ${ }^{2}$
}

To cite: Barrett S, Begg S, 0'Halloran P, et al. Costeffectiveness of telephone coaching for physically inactive ambulatory care hospital patients: economic evaluation alongside the Healthy $4 \mathrm{U}$ randomised controlled trial. BMJ Open 2019;9:e032500. doi:10.1136/ bmjopen-2019-032500

- Prepublication history and additional material for this paper are available online. To view these files, please visit the journal online (http://dx.doi. org/10.1136/bmjopen-2019032500).

Received 21 June 2019 Revised 31 October 2019 Accepted 25 November 2019

Check for updates

(C) Author(s) (or their employer(s)) 2019. Re-use permitted under CC BY-NC. No commercial re-use. See rights and permissions. Published by BMJ.

For numbered affiliations see end of article.

\section{Correspondence to}

Prof Michael Kingsley, Holsworth Research Initiative, La Trobe University, Bendigo, Victoria ,Australia;

M.Kingsley@latrobe.edu.au

\section{ABSTRACT}

Objective To assess whether telephone coaching is a cost-effective method for increasing physical activity and health-related quality of life for insufficiently active adults presenting to an ambulatory care clinic in a public hospital. Design An economic evaluation was performed alongside a randomised controlled trial.

Setting Participants were recruited from an ambulatory care clinic in a public hospital in regional Australia. Participants Seventy-two adults (aged 18-69) deemed insufficiently physically active via self-report.

Interventions Participants were randomised to either an intervention group that received an education session and eight sessions of telephone coaching over a 12-week period, or to a control group that received the education session only. The intervention used in the telephone coaching was integrated motivational interviewing and cognitive behavioural therapy.

Outcome measures The primary health outcome was change in moderate-to-vigorous physical activity (MVPA), objectively measured via accelerometry. The secondary outcome was the quality-adjusted life-year (QALY) determined by the 12-item Short Form Health Survey Questionnaire. Outcome data were measured at baseline, postintervention (3 months) and follow-up (6 months). Incremental cost-effectiveness ratios (ICERs) were calculated for each outcome. Non-parametric bootstrapping techniques and sensitivity analyses were performed to account for uncertainty.

Results The mean intervention cost was $\$ 279 \pm \$ 13$ per person. At 6 months follow-up, relative to control, the intervention group undertook 18 more minutes of daily MVPA at an ICER of $\$ 15 /$ min for each additional minute of MVPA. With regard to QALYs, the intervention yielded an ICER of $\$ 36857$ per QALY gained. Sensitivity analyses indicated that results were robust to varied assumptions.

Conclusion Telephone coaching was a low-cost strategy for increasing MVPA and QALYs in insufficiently physically active ambulatory care hospital patients. Additional research could explore the potential economic impact of the intervention from a broader healthcare perspective.

Trial registration number ANZCTR: ACTRN12616001331426.
Strengths and limitations of this study

- This study is the first to investigate the costeffectiveness of telephone delivered motivational interviewing and cognitive behavioural therapy for insufficiently physically active secondary care hospital patients.

- Economic evaluations enable hospitals to estimate the costs of delivering preventive health interventions, which are important for addressing the burden of chronic disease.

- Changes in physical activity were measured using accelerometers, strengthening the confidence in the findings.

- Results are limited by a short time horizon and a narrow costing perspective.

- Further research is needed to explore the potential long-term economic impact of the intervention from a broader healthcare perspective.

\section{INTRODUCTION}

Insufficient physical activity (PA) is an established risk factor for the development of a number of chronic diseases, including cardiovascular disease, type 2 diabetes and obesity. ${ }^{1}$ Despite the well-established benefits of PA, ${ }^{2}$ more than half of the population does not attain sufficient levels of PA to derive such benefits. $^{3}$ The estimated cost of insufficient PA in Australia is \$A805 million per annum. ${ }^{4}$ Addressing the prevalence of insufficient PA is a major public health priority, and necessitates that multiple sectors of the healthcare industry are actively engaged in PA promotion..$^{5}$

Hospitals are important settings in which to offer health promotion interventions, for both admitted and ambulatory care. In the hospital setting, ambulatory care refers to non-admitted clinics that patients attend for specialist medical care. Patients attending 
ambulatory care hospital clinics are more likely than the general population to have one or more chronic disease. ${ }^{67}$ Hospital patients can be motivated to engage with lifestyle behaviour change as their health is already compromised. ${ }^{89}$ Attending a hospital has been identified as a major life event, ${ }^{10}$ and a hospital visit has the potential to initiate health behaviour change. ${ }^{8}$ Ambulatory care settings provide an ideal opportunity for behaviour change interventions. ${ }^{8}{ }^{10}$ Substantial efforts have been made to promote increased engagement in PA using individual and population-based approaches. ${ }^{11}$ This has resulted in an increased use of behaviour change interventions to influence participation in PA. ${ }^{12}$

A number of studies suggest that telephone coaching results in improved clinical outcomes, self-efficacy and health status, ${ }^{13} 14$ as well as increases in PA. ${ }^{15} 16$ Additional work is required to embed telephone coaching within existing health services. ${ }^{13} 15$ To make the benefits of telephone coaching more broadly available for hospital outpatients, telephone coaching has been delivered in addition to standard ambulatory care. ${ }^{17}$ The addition of telephone coaching to standard care resulted in significant improvements in objectively measured PA and health-related outcomes; ${ }^{17}$ however, the cost to improve these outcomes has not been reported.

Few studies have employed any form of economic analyses on telephone coaching, and little is known about the relative cost-effectiveness of adding telephone coaching to routine care in ambulatory care hospital settings. The purpose of this study was to evaluate the cost-effectiveness of the Healthy $4 \mathrm{U}$ programme for increasing measured PA and the number of quality-adjusted life-years (QALYs) experienced over a 6-month period from a hospital perspective.

\section{METHODS}

\section{Study design}

The Healthy4U study was a single-blind randomised controlled trial (RCT) reported in line with the CHEERS reporting guidelines ${ }^{18}$ (online supplementary file 1 ). The trial design, participants, sample size, intervention, outcomes and ethics approvals have been described in detail elsewhere. ${ }^{17}$ Briefly, between October 2016 and December 2017, 72 insufficiently physically active adults, aged 18-69 years, were recruited from ambulatory care clinics at a major hospital in a regional town in Victoria, Australia. The primary aim was to promote change in objectively measured PA during the trial.

\section{Intervention}

All enrolled participants attended a 30-min group education session. The education session was a facilitated learning session focused on self-management and lifestyle modification, and was carried out using a selfdetermination theory framework. ${ }^{19}$

The intervention group completed a telephone-based, integrated motivational interviewing and cognitive behavioural therapy (MI-CBT) intervention, delivered in eight 30 -min sessions over 12 weeks. The intervention was delivered by an experienced allied health clinician trained in MI-CBT. All participants enrolled into the control arm attended the education session. Apart from contact regarding follow-up outcome measures, participants in the control group received no further contact initiated by the research team.

\section{Measurement of effects}

Outcome measures were recorded at baseline, after 3 months of intervention (postintervention) and at 6 months (follow-up) by assessors blinded to the study group assignment. The primary outcome measure was change in moderate-to-vigorous physical activity (MVPA), objectively measured by accelerometry (wGT3X-BT; Actigraph, USA). Daily MVPA was determined using the manufacturers software (Actilife; Actigraph, USA) and the Freedson Adult (1998) cut point (vector magnitude $>1961 \mathrm{cpm}) .{ }^{20}$ To be included in the analysis, a minimum wear time of $\geq 10$ hour/day for 5 of the 7-day period was required, including at least one weekend day. ${ }^{21}$ Weekly PA totals were summed from the daily totals for persons with 7 valid days of monitoring, or estimated as seven times the average daily total for persons with 5-6 valid days of monitoring. Using the summed weekly totals, participants were classified as either meeting or not meeting the recommended PA guidelines. ${ }^{22}$

A secondary outcome was a change in health-related quality of life (HrQoL) and QALYs, which was derived from the Medical Outcomes Study Short Form 12 Health Survey (SF-12) and the standard Brazier algorithm. ${ }^{23} 24$ The SF-12 scores were converted to utility scores on a scale of $0-1$, with a higher score indicating a more favourable health state. ${ }^{24}$ These utility estimates were converted to QALYs by calculating the 'area under the curve' utility estimates for the different follow-up time intervals for each participant, weighted by the length of follow-up at that time interval.

\section{Measurement of costs}

The cost analysis was designed and conducted from a hospital perspective, which allows healthcare organisations to gauge the approximate cost of offering this programme. ${ }^{25} \mathrm{~A}$ bottom-up microcosting approach was used to calculate the intervention costs. ${ }^{26}$ This approach involves the detailed collection of information regarding the quantities of resources consumed while implementing and executing the interventions, as well as their respective unit prices. ${ }^{26}$ Only those costs involved in implementing the intervention (eg, training of individuals carrying out and undertaking the intervention) were included. Protocol-driven costs, namely the costs of gathering data as part of the clinical trial were considered to be sunk costs and were therefore excluded from the cost-effectiveness analysis. ${ }^{25}$

The programme costs included group facilitator time, intervention time and supplies. The group facilitator's 
time was spent preparing for and facilitating group meetings. The intervention assistant's time was calculated as the time spent undertaking reminder phone calls to participants. The intervention costs were calculated as the time spent in one-to-one consultation with the participants. Both group facilitation and intervention costs were calculated using the annual salary of an experienced allied health clinician as they would most likely be used in delivering MI-CBT were the intervention to be implemented on a large scale (\$A82 924). Intervention assistant costs were based on the annual salary of an Allied Health Assistant (\$A45 338). Finally, the costs of supplies, including the programme manuals, were included in the programme cost.

The group facilitator's time per group meeting was estimated at 2.5 hours, which included 0.5 hours for the group meeting itself, 1.0 hour to set up before and clean up after the group meeting, and 1.0 hour to prepare for the group meeting (ie, reviewing meeting notes and presentation material). The group facilitator's cost per meeting per participant was calculated by dividing the facilitator's cost per meeting by the number of participants who attended each meeting. Due to the short timeframe in which costs and effects occurred, discounting was not necessary. ${ }^{27}$ All programme costs were calculated in 2017 Australian dollars (AU\$).

\section{Statistical analysis}

Analyses of trial data have been reported elsewhere. ${ }^{17}$ In brief, mixed-model analysis of variances were used to assess the effects of the intervention on each of the outcome variables. The mean $\pm \mathrm{SD}$ for the overall cost and for the change in each outcome at 6 months was calculated. For each outcome, the incremental costeffectiveness ratio (ICER) was calculated by dividing the difference in costs by the difference in effects between the intervention and control groups. The difference in effects between the two groups was calculated using a change from baseline approach to control for different baseline utilities. Uncertainty in the ICER estimates was accounted for by generating 1000 bootstrap replicates of the data set, a widely used method in health economic evaluations. Probabilistic sensitivity analysis was completed by calculating the cost-effectiveness acceptability curve (CEAC) derived from the bootstrap replicates. CEAC indicates the probability that the intervention was cost effective at different values of willingness to pay for the additional improvement in the outcome. ${ }^{28}$

In addition, four sensitivity analyses were used to examine how the results changed under different input assumptions. In the first two analyses, personnel wages and training costs were varied by $20 \%$ in either direction before recalculating the ICERs. The third sensitivity analysis used the summed weekly MVPA totals to estimate the ICER for each additional minute of MVPA per week. The fourth sensitivity analysis considered a different outcome measure for PA, using the summed weekly MVPA totals to estimate the ICER for changing one individual from insufficiently physically active to sufficiently physically active ( $\geq 150$ min MVPA per week). ${ }^{22}$

\section{Patient and public involvement}

Patients were not involved in the research question, study design or the conduction of the study.

\section{RESULTS}

\section{Resource use and costs}

Seventy-two participants were randomised; the group consisting of 54 females and 18 males, with an average age of $53 \pm 8$ years (online supplementary file 2). A total of 72 participants completed their baseline and 3-month assessment, and 68 participants completed the 6-month assessment. For participants with missing data at 6-month follow-up ( $\mathrm{n}=2$ in both groups), the last-observation-carried forward approach was adopted. The programme resources and cost per participant are described in table 1 . Attendance at the education session

Table 1 Utilisation and cost of programme delivery for intervention and control groups

\begin{tabular}{|c|c|c|c|c|c|}
\hline Item & Provider & Units & $\begin{array}{l}\text { Time } \\
\text { (hours) }\end{array}$ & Cost/hour \$A & Total \$A cost per participant \\
\hline \multicolumn{6}{|l|}{ Intervention group } \\
\hline Phone call reminders & Intervention assistant & 8 & 0.25 & 22.80 & 45.6 \\
\hline Intervention sessions & Allied health professional & 8 & 0.5 & 41.90 & 167.6 \\
\hline Total cost/participant & & & & & 279 \\
\hline \multicolumn{6}{|l|}{ Control group } \\
\hline Group sessions & Allied health professional & 1 & 2.5 & 41.90 & 17.45 \\
\hline Programme manual & & & & & 3.5 \\
\hline
\end{tabular}


Table 2 Costs, changes in outcomes and incremental cost-effectiveness ratios at follow-up

\begin{tabular}{|c|c|c|c|c|c|}
\hline Outcome & Cost\$A/participant & Outcome & Incremental cost \$A & Incremental outcome & ICER \\
\hline \multicolumn{6}{|l|}{ MVPA } \\
\hline Control & 21 & 23 & & & \\
\hline \multicolumn{6}{|l|}{ QALYs } \\
\hline Control & 21 & -0.005 & & & \\
\hline
\end{tabular}

ICER, incremental cost-effectiveness ratio; MVPA, moderate-to-vigorous physical activity; QALYs, quality-adjusted life-years.

was mandatory, with full attendance recorded for both groups. The mean group programme time was $34 \pm 6 \mathrm{~min}$, and the mean total participant time spent in the intervention was $242 \pm 14 \mathrm{~min}$. The mean cost per participant was $\$ 279 \pm \$ 10$ for intervention versus $\$ 21 \pm \$ 3$ for control. The main component costs of MI-CBT were intervention delivery and training.

\section{Health outcomes}

Table 2 presents the mean costs and the mean change in each outcome at follow-up for each group, and the corresponding ICERs. The ICER for MVPA was $\$ 15$ per each additional minute of MVPA per day. The difference in QALYs between intervention and control groups was 0.007 QALYs over the course of the follow-up period. The ICER for the intervention group in comparison with the control group was $\$ 36857$ per QALY gained. Figures 1 and 2 illustrate the CEAC for each outcome derived from non-parametric bootstrapping replicates. For PA, given a willingness to pay of $\$ 15$ per additional minute of MVPA, the probability that the intervention was cost effective was $67 \%$ (figure 1). At a willingness to pay of $\$ 37000$ per QALY gained, the probability that the intervention is cost effective was $52 \%$ (figure 2). If the decision maker was willing to pay $\$ 40000$ per QALY, the probability of costeffectiveness for the intervention was $70 \%$ (figure 2).

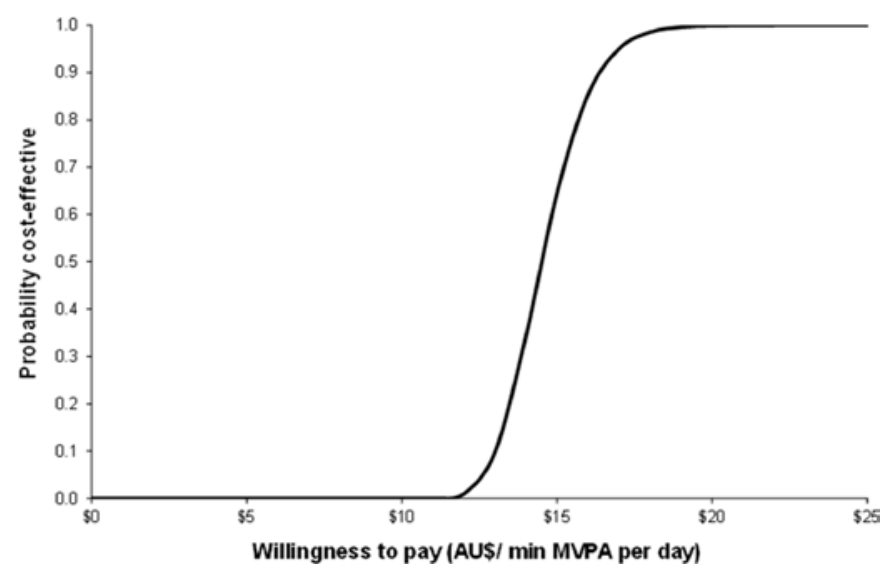

Figure 1 Cost-effectiveness acceptability curve showing the probability of the interventions being cost-effective in comparison to control for moderate-to-vigorous physical activity (MVPA).
In the sensitivity analyses, training and implementation costs were varied $20 \%$ in each direction, and the corresponding ICERs were recalculated (table 3 ). The varied ICERs for MVPA were found to range from $\$ 11$ to $\$ 17$ per each additional minute of MVPA per day, while the ICER for QALYs ranged from \$29 428 to AU\$44 285 per QALY gained. The third sensitivity analysis demonstrated an ICER of \$2.86 per additional minute of MVPA per week. The final sensitivity analysis found that the intervention group was $33 \%$ more likely than control to meet recommended PA guidelines at follow-up, with an ICER of $\$ 781$ per PA guideline attained.

\section{DISCUSSION}

This study examined the clinical and economic implications of a behaviour change intervention for changes in PA and QALYs for insufficiently physically active adults presenting to an ambulatory care clinic in a regional public hospital setting. Over the follow-up period, the MI-CBT intervention was significantly more effective than control in increasing PA and HrQoL. To the best of our knowledge, this is the first study to evaluate the cost-effectiveness and cost-utility of an integrated MI-CBT intervention for health-related behaviour change.

Telephone coaching can be cost effective for increasing PA with patients with or at risk of chronic disease. ${ }^{29} 30$

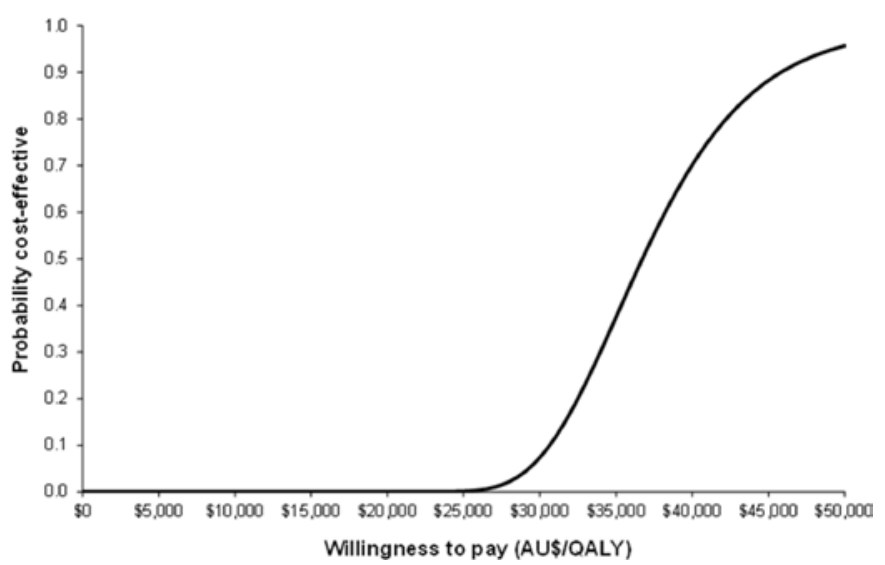

Figure 2 Cost-effectiveness acceptability curve showing the probability of the interventions being cost-effective in comparison to control for quality-adjusted life-years (QALYs). 
Table 3 Sensitivity analyses for costs, changes in outcomes and incremental cost-effectiveness ratios at follow-up

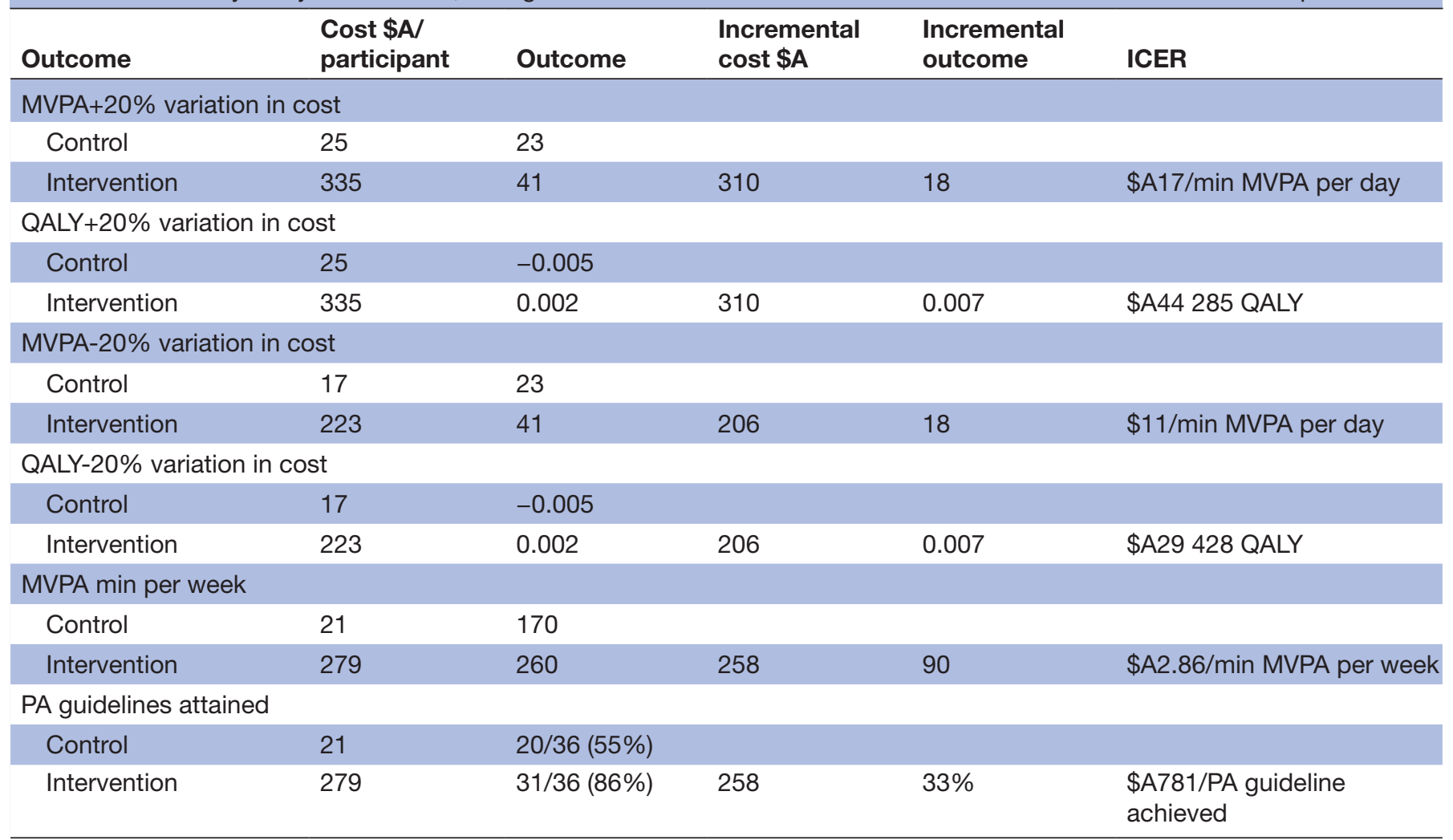

ICER, incremental cost-effectiveness ratio; MVPA, moderate-to-vigorous physical activity; PA, physical activity; QALYs, quality-adjusted lifeyears.

However, little is known about the cost-effectiveness of adding preventive interventions to routine hospital care, where implementing an intervention requires an upfront investment of money. The total estimated cost of delivering the MI-CBT intervention was $\$ 279$ per person, resulting in the average attainment of $41 \pm 12$ min of MVPA per day at follow-up. The per person cost was similar to the $\$ 245$ per person found in a recent primary care intervention for PA change, ${ }^{31}$ while both are considerably lower than costs of $\$ 1756 /$ person $^{32}$ and $\$ 1562 /$ person $^{33}$ reported in other lifestyle interventions aiming at changes in PA.

The cost-effectiveness analysis for measured MVPA indicated a cost of $\$ 15$ per each additional minute of MVPA per day. Over the 6-month follow-up period, this can be translated to a total cost of $\$ 9$ per day, or $\$ 63$ per week to increase MVPA by $150 \mathrm{~min}$. Sensitivity analysis indicated a one-time cost of $\$ 2.86$ per each additional minute of MVPA per week, or $\$ 8.25$ per week to increase MVPA to 150 min a week over the 6-month follow-up. The cost of $\$ 8.25$ per week to increase MVPA to $150 \mathrm{~min}$ is comparable to the $\$ 4.99,,^{34} \$ 8.13^{35}$ and $\$ 10.19^{36}$ per week found in other interventions aimed at increasing PA. Increases in PA result in decreased healthcare use, even in the short term, which result in net savings to society over time. ${ }^{37}$ Determining the cost-effectiveness of integrating telephone coaching into routine care from a hospital perspective is dependent on the willingness-to-pay for each additional minute of MVPA. Interpreting the ICER of $\$ 15$ per additional minute of MVPA found here is difficult as there is no standard value for how much policy-makers are willing to pay per additional minute of MVPA. ${ }^{34}$ The CEAC indicated a probability of $67 \%$ that $\$ 15$ per additional minute of MVPA was cost-effective (figure 1).

The intervention group was $33 \%$ more likely than the control group to undertake sufficient PA at follow-up. Sensitivity analysis demonstrated an ICER of $\$ 781$ for converting one insufficiently physically active adult to a sufficiently active state over the 6-month follow-up period. This value falls with the ranges of $\$ 175$ to $\$ 1801,38$ and $\$ 521$ to $\$ 5790^{39}$ estimated in systematic reviews investigating the cost-effectiveness of PA interventions. Undertaking sufficient PA is strongly associated with decreased risk of chronic disease, morbidity and mortality, ${ }^{1}$ as well as decreased healthcare expenditure over time. ${ }^{37}$

In the cost-utility analysis, an incremental change in QALYs of 0.007 was demonstrated between the intervention and control groups, resulting in an ICER of $\$ 36857$ per QALY gained. The ICER of \$36 857 per QALY gained is considerably smaller than the \$58 924 per QALY gained ${ }^{32}$ and the $\$ 68101$ per QALY gained $^{33}$ found in similar intervention studies. Oksman et al recently reported an ICER of $\$ 48000$ per QALY gained for a telephone-based health coaching intervention for chronic disease patients. ${ }^{40}$ Direct comparison with the results from the aforementioned studies is challenging because different cost perspectives were considered in 
these analyses. Nonetheless, the ICER of $\$ 36857$ per QALY falls under the commonly used threshold of $\$ 50$ 000 per QALY gained proposed for medical treatments and procedures. ${ }^{41}$ Sensitivity analysis demonstrated that costs for the Healthy4U study are only mildly sensitive to typical variation in the cost input values. A variation in hourly wage costs had the largest impact on the ICERs; however, the ICERs remained well below the threshold of $\$ 50000$ per QALY gained at all imputed values. The CEAC provided a probability of $52 \%$ that the intervention was cost effective at a willingness to pay of $\$ 37000$ per QALY gained (figure 2).

Behaviour change interventions are typically used to modify specific lifestyle factors known to predispose individuals to increased risk of chronic disease over the longer term. ${ }^{42}$ The long-term impact of such interventions on overall quality of life is less established. While the follow-up period in this study was too short for the mediating effect of PA on broader health outcomes to become fully apparent, ${ }^{42}$ the relatively small observed change in QALYs over 6 months was a combination of the -0.005 fall in the control group and the 0.002 increase in the intervention group. This finding supports the suggestion that an important impact of behavioural interventions on quality-of-life over the longer term might be to attenuate expected declines in HrQoL. ${ }^{43}$

This study was unique in that we enrolled participants from an ambulatory care clinic in a public hospital, integrating preventative health into secondary care. It is important to note that this intervention was carried out in addition to standard care, not as a substitution, and as such an economic evaluation costed from a hospital perspective offers healthcare providers an estimate of the costs and effects of adding a preventative health intervention to clinical care. This study is one of the few economic evaluations of telephone coaching carried out in real-life settings using an RCT design. The addition of preventive health measures is likely to cost hospitals more; however, these preventive measures might be worthwhile due to the substantial health benefits that they confer, relative to their cost. ${ }^{37}$ Nevertheless, implementation remains a challenge for hospitals, ${ }^{15}$ and it will be important to engage with key stakeholders, especially clinic leaders, to identify specific patients who can benefit from telephone coaching. ${ }^{44}$ Health services with high implementation rates of telephone coaching have used multicomponent strategies to engage staff, as they were the most important source of referrals. ${ }^{44}$ Investigating hospital clinicians' practice and beliefs around preventive health can also facilitate the development of pathways to increase preventive health practice in the hospital setting. ${ }^{45}$

The greatest cost of the intervention was in the delivery, due to the fact that it was delivered individually by trained personnel. These costs could be reduced by decreasing the number of intervention sessions, with a recent metaanalysis indicating that five sessions of MI-CBT are significantly effective for PA change. ${ }^{46}$ Costs could potentially be reduced by incorporating digital technology into the intervention to decrease the time spent by trained professionals in 1:1 sessions. Innovations in digital technologies can assist individuals with health behaviour change, ${ }^{47}$ which can potentially reduce healthcare expenditure. ${ }^{47} 48$ The long-term cost-effectiveness of digital health technologies has not been established. ${ }^{47}$

The use of objectively measured PA at all time points was a considerable strength of the study. Objective measures offer more precise estimates of activity intensity while removing many of the issues associated with participant recall and response bias. ${ }^{49}$ Individuals have been demonstrated to overestimate their PA levels via self-report. ${ }^{38}$ Overestimation of PA can result in inaccurate estimations of both effectiveness and the cost-effectiveness of interventions. ${ }^{50}$ Using objectively measured changes in PA and the collection of full cost data for all participants strengthens our findings. ${ }^{49}$

This study has a number of limitations. A noteworthy limitation of this study is the restricted perspective used for the economic evaluation. Using a single hospital perspective might have led to the exclusion of important costs and benefits from a societal perspective, including healthcare utilisation and changes in productivity. ${ }^{51}$ Economic analyses from a societal perspective offer the most comprehensive evidence from which to base decisions. ${ }^{51}$ However, due to the relatively short follow-up time of this study, it was not feasible to undertake this method. Significant effects on overall healthcare utilisation or productivity loss were not expected over the timeframe of this study. ${ }^{52}$ As the intervention was delivered using the telephone, the intervention required relatively small amounts of participant time. With this in mind, the participant opportunity costs were expected to be small and therefore not included in the analyses. Additionally, the study participants included 54 females and 18 males, which might limit the generalisability of the current findings to different populations.

To the best of our knowledge, this is the first study comparing the cost-effectiveness and cost-utility of an integrated MI-CBT intervention for health behaviour change among community-dwelling adults presenting to a secondary care clinic in a regional public hospital setting. Considering the large group of people who might benefit from such an intervention (ie, approximately $50 \%$ of adults aged 18-69 years in Australia who are currently insufficiently physically active), the widespread adoption and implementation of MI-CBT to increase PA could have important economic implications. However, further research with a more comprehensive economic analysis is needed to investigate whether the long-term benefits of MI-CBT might justify this type of investment.

\section{CONCLUSION}

The Healthy $4 \mathrm{U}$ programme is a relatively low-cost strategy for increasing PA among insufficiently physically active adults presenting to an ambulatory care hospital clinic. The intervention increased measured PA and quality 
of life at low costs, with positive effects maintained out to 6 months. By increasing PA and quality of life at low costs, integrating telephone coaching into secondary hospital care offers a potentially cost-effective investment to produce better public health outcomes. The results are however grounded on a short-term follow-up and a restricted economic perspective, and more evidence is needed to explore the potential long-term economic impact of the intervention from a broader healthcare perspective.

\section{Author affiliations}

${ }^{1}$ Health Promotion, Bendigo Health - Bendigo Hospital Campus, Bendigo, Victoria, Australia

${ }^{2}$ La Trobe Holsworth Research Initiative, La Trobe University, Bendigo, Victoria, Australia

${ }^{3}$ School of Psychology and Public Health, La Trobe University, Bundoora, Victoria, Australia

\section{Twitter Stephen Barrett @barrettstephen1}

Contributors SBa, MK, SBe and PO'H conceived the project and assisted with the protocol design. SBa managed the trial including recruitment and data collection, coordinated the intervention program, performed statistical analysis and drafted the manuscript. SBa and SBe interpreted the data and drafted the manuscript. All authors read, edited and approved the final manuscript as submitted.

Funding The authors have not declared a specific grant for this research from any funding agency in the public, commercial or not-for-profit sectors.

Competing interests None declared.

Patient consent for publication Not required.

Ethics approval The study was approved by the Research Ethics Committees of Bendigo Health Care group (approved 16 September 2016; reference number LNR/16/BHCG/42) and La Trobe University College of Science Health and Engineering Human Ethics Sub-Committee (approved 3 October 2016).

Provenance and peer review Not commissioned; externally peer reviewed.

Data availability statement Data are available upon reasonable request.

Open access This is an open access article distributed in accordance with the Creative Commons Attribution Non Commercial (CC BY-NC 4.0) license, which permits others to distribute, remix, adapt, build upon this work non-commercially, and license their derivative works on different terms, provided the original work is properly cited, appropriate credit is given, any changes made indicated, and the use is non-commercial. See: http://creativecommons.org/licenses/by-nc/4.0/.

ORCID iD

Stephen Barrett http://orcid.org/0000-0003-2505-733X

\section{REFERENCES}

1 Warburton DER, Nicol CW, Bredin SS. Health benefits of physical activity: the evidence. Can Med Assoc J 2006;174:801-9.

2 Khan KM, Thompson AM, Blair SN, et al. Sport and exercise as contributors to the health of nations. Lancet 2012;380:59-64.

3 World Health Organization. Global health risks: mortality and burden of disease attributable to selected major risks. World Health Organization, 2009. http://www.who.int/iris/handle/10665/44203

4 Ding D, Lawson KD, Kolbe-Alexander TL, et al. The economic burden of physical inactivity: a global analysis of major non-communicable diseases. Lancet 2016;388:1311-24.

5 Sparling PBet al. Promoting physical activity: the new imperative for public health. Health Educ Res 2000;15:367-76.

6 Britt HC, Harrison CM, Miller GC, et al. Prevalence and patterns of multimorbidity in Australia. Med J Aust 2008;189:72-7.

7 Steiner CA, Friedman B. Hospital utilization, costs, and mortality for adults with multiple chronic conditions, nationwide inpatient sample, 2009. Prev Chronic Dis 2013;10:E62.

8 Gate L, Warren-Gash C, Clarke A, et al. Promoting lifestyle behaviour change and well-being in hospital patients: a pilot study of an evidence-based psychological intervention. $J$ Public Health 2016;38:e292-300.

9 Lawson PJ, Flocke SA. Teachable moments for health behavior change: a concept analysis. Patient Educ Couns 2009;76:25-30.

10 Allender S, Hutchinson L, Foster C. Life-change events and participation in physical activity: a systematic review. Health Promot Int 2008;23:160-72.

11 Foster C, Hillsdon M, Thorogood M, et al. Interventions for promoting physical activity. Cochrane Database Syst Rev 2005;18.

12 Stephens J, Allen J. Mobile phone interventions to increase physical activity and reduce weight: a systematic review. $J$ Cardiovasc Nurs 2013;28:320-9.

13 Dennis SM, Harris M, Lloyd J, et al. Do people with existing chronic conditions benefit from telephone coaching? a rapid review. Aust Health Rev 2013;37:381-8.

14 Kivelä K, Elo S, Kyngäs $\mathrm{H}$, et al. The effects of health coaching on adult patients with chronic diseases: a systematic review. Patient Educ Couns 2014;97:147-57.

15 Eakin EG, Lawler SP, Vandelanotte C, et al. Telephone interventions for physical activity and dietary behavior change. Am J Prev Med 2007;32:419-34.

16 O'Hara BJ, Phongsavan P, Venugopal K, et al. Effectiveness of Australia's get healthy information and coaching Service ${ }^{\circledR}$ : translational research with population wide impact. Prev Med 2012;55:292-8

17 Barrett S, Begg S, O'Halloran P, et al. Integrated motivational interviewing and cognitive behaviour therapy can increase physical activity and improve health of adult ambulatory care patients in a regional Hospital: the Healthy $4 \mathrm{U}$ randomised controlled trial. BMC Public Health 2018;18:1166.

18 Husereau D, Drummond M, Petrou S, et al. Consolidated health economic evaluation reporting standards (cheers) statement. BMJ 2013;346.

19 Vansteenkiste M, Sheldon KM. There's nothing more practical than a good theory: integrating motivational interviewing and selfdetermination theory. Br J Clin Psychol 2006;45:63-82.

20 Freedson PS, Melanson E, Sirard J. Calibration of the computer science and applications, Inc. accelerometer. Medicine \& Science in Sports \& Exercise 1998;30:777-81.

21 Olds T, Ferrar KE, Gomersall SR, et al. The elasticity of time. Health Educ Behav 2012;39:732-6.

22 Nelson ME, Rejeski WJ, Blair SN, et al. Physical activity and public health in older adults: recommendation from the American College of sports medicine and the American heart association. Circulation 2007;116:1094-105.

23 Ware Jr JE, Kosinski M, Keller SD. A 12-Item short-form health survey: construction of scales and preliminary tests of reliability and validity. Med Care 1996;34:220-33.

24 Brazier J, Roberts J, Deverill M. The estimation of a preferencebased measure of health from the SF-36. J Health Econ 2002;21:271-92.

25 Drummond M, Sculpher M, Torrance G, et al. Methods for the economic evaluation of health care programmes. 3rd edn. New York: Oxford University Press, 2005.

26 Tan SS, Rutten FFH, van Ineveld BM, et al. Comparing methodologies for the cost estimation of hospital services. Eur $J$ Health Econ 2009;10:39-45.

27 Glick HA, Doshi JA, Sonnad SS, et al. Economic evaluation in clinical trials. Oxford: Oxford University Press, 2014.

28 Appleby J, Devlin N, Parkin D. NICE's cost effectiveness threshold. BMJ 2007;335:358-9.

29 Hutchison AJ, Breckon JD. A review of telephone coaching services for people with long-term conditions. J Telemed Telecare 2011;17:451-8.

30 Varney JE, Liew D, Weiland TJ, et al. The cost-effectiveness of hospital-based telephone coaching for people with type 2 diabetes: a 10 year modelling analysis. BMC Health Serv Res 2016;16:521.

31 Ewald B, Stacey F, Johnson N, et al. Physical activity coaching by Australian Exercise Physiologists is cost effective for patients referred from general practice. Aust N Z J Public Health 2018;42:12-15.

32 Groessl EJ, Kaplan RM, Castro Sweet CM, et al. Cost-effectiveness of the life physical activity intervention for older adults at increased risk for mobility disability. GERONA 2016;71:656-62.

33 Groessl EJ, Kaplan RM, Blair SN, et al. A cost analysis of a physical activity intervention for older adults. $J$ Phys Act Health 2009;6:767-74.

34 Johnson TJ, E Schoeny M, Fogg L, et al. The cost of increasing physical activity and maintaining weight for midlife sedentary African American women. Value Health 2016;19:20-7. 
35 Elley CR, Garrett S, Rose SB, et al. Cost-effectiveness of exercise on prescription with telephone support among women in general practice over 2 years. Br J Sports Med 2011;45:1223-9.

36 Sevick MA, Napolitano MA, Papandonatos GD, et al. Costeffectiveness of alternative approaches for motivating activity in sedentary adults: results of Project STRIDE. Prev Med 2007;45:54-61.

37 Martinson BC, Crain AL, Pronk NP, et al. Changes in physical activity and short-term changes in health care charges: a prospective cohort study of older adults. Prev Med 2003;37:319-26.

38 GC V, Wilson ECF, Suhrcke M, et al. Are brief interventions to increase physical activity cost-effective? A systematic review. $\mathrm{Br} J$ Sports Med 2016;50:408-17.

39 Garrett S, Elley CR, Rose SB, et al. Are physical activity interventions in primary care and the community cost-effective? A systematic review of the evidence. Br J Gen Pract 2011;61:e125-33.

40 Oksman E, Linna M, Hörhammer I, et al. Cost-effectiveness analysis for a tele-based health coaching program for chronic disease in primary care. BMC Health Serv Res 2017;17:138.

41 Hirth RA, Chernew ME, Miller E, et al. Willingness to pay for a quality-adjusted life year: in search of a standard. Med Decis Making 2000;20:332-42.

42 Goyder E, Hind D, Breckon J, et al. A randomised controlled trial and cost-effectiveness evaluation of 'booster' interventions to sustain increases in physical activity in middle-aged adults in deprived urban neighbourhoods. Health Technol Assess 2014;18:1.

43 Cutler DM. Behavioral health interventions: what works and why. In: Critical perspectives on racial and ethnic differences in health in late life, 2004: 643.

44 Damschroder LJ, Reardon CM, Sperber N, et al. Implementation evaluation of the telephone lifestyle coaching (TLC) program: organizational factors associated with successful implementation. Transl Behav Med 2017;7:233-41.

45 Barrett S, Begg S, Sloane A, et al. Surgeons and preventive health: a mixed methods study of current practice, beliefs and attitudes influencing health promotion activities amongst public hospital surgeons. BMC Health Serv Res 2019;19:358.

46 Barrett S, Begg S, O'Halloran P, et al. Integrated motivational interviewing and cognitive behaviour therapy for lifestyle mediators of overweight and obesity in community-dwelling adults: a systematic review and meta-analyses. BMC Public Health 2018;18:1160.

47 Sanyal C, Stolee P, Juzwishin D, et al. Economic evaluations of eHealth technologies: a systematic review. PLoS One 2018:13:e0198112.

48 Jiang X, Ming W-K, You JHS. The cost-effectiveness of digital health interventions on the management of cardiovascular diseases: systematic review. J Med Internet Res 2019;21:e13166.

49 Prince SA, Adamo KB, Hamel M, et al. A comparison of direct versus self-report measures for assessing physical activity in adults: a systematic review. Int J Behav Nutr Phys Act 2008;5.

50 Bize R, Johnson JA, Plotnikoff RC. Physical activity level and healthrelated quality of life in the general adult population: a systematic review. Prev Med 2007;45:401-15.

51 Jönsson B. Ten arguments for a societal perspective in the economic evaluation of medical innovations. Eur J Health Econ 2009;10:357-9.

52 van Keulen HM, Bosmans JE, van Tulder MW, et al. Costeffectiveness of tailored print communication, telephone motivational interviewing, and a combination of the two: results of an economic evaluation alongside the Vitalum randomized controlled trial. Int $J$ Behav Nutr Phys Act 2010;7. 